\title{
Newcomer Corner
}

\section{Vanessa Klein, Hamburg Gemeinsam einsam: Phånomen Phubbing \\ Untersuchungen zur unangebrachten Smartphone-Nutzung im privaten und öffentlichen Miteinander}

Phubbing - das ist die unangebrachte Smartphone-Nutzung im privaten und öffentlichen Miteinander. Das Kofferwort aus den englischen Begriffen phone und snubbing bezeichnet das verbreitete Verhalten, sich auch im Beisein anderer Personen mit dem Smartphone zu beschäftigen. Das Ziel der Studie zum Thema war es Ursachen für die unangemessene Smartphone-Nutzung zu ermitteln und zu klären, ob Phubbing eine Beeinträchtigung des Kommunikationsverhaltens und des gesellschaftlichen Miteinanders darstellt. Hierfür wurde eine Onlinebefragung unter Studierenden des Departments Information an der HAW Hamburg durchgeführt, in der Erfahrungswerte und Meinungen der Teilnehmer zu Phubbing eingeholt wurden. Ergänzt wurde sie durch eine Beobachtungsstudie von realen Phubbing-Situationen an öffentlichen Plätzen. Die Erkenntnisse aus den genannten empirischen Untersuchungen bildeten die Grundlage für ein Fokusgruppenprojekt mit ausgewählten Teilnehmern der Onlinebefragung, in dessen Rahmen die Befunde tiefergehend diskutiert und analysiert wurden. Die Studie ist in dem Zeitraum von November 2013 bis März 2014 entstanden.

Deskriptoren: Mobilkommunikation; Öffentlich, Benutzerforschung, Bevölkerung, Soziales Netz, Informationsverhalten, Empirische Untersuchung, Befragung; Online

\section{Collective solitude: Phenomenon Phubbing. Analysis of the inappropriate use of smartphones in private and public communication}

Phubbing - that is the inappropriate use of smartphones in private and public communication. It is a portmanteau derived from the English terms phone and snubbing, and describes the behaviour of occupying oneself with a smartphone while interacting with other people. The study goal was both to identify reasons for the mistimed smartphone-use and to find out if phubbing affects the ability to communicate and socially interact. An online survey of students of the Department of Information at the HAW Hamburg allowed detecting empirical values and personal opinions concerning the topic of phubbing. Furthermore, an observational study captured several real phubbing-situations and reactions in public places. A focus group project with selected participants of the online survey finally discussed and analysed the results of the preceding empirical methods in detail. The study took place from November 2013 to March 2014.

Descriptors: Mobile communication; Online, User analysis, Population, Social network, Information-seeking behaviour, Empirical study, Inquiry; Online

\section{Ensemble et pourtant isolés: le phénomène du phubbing : enquêtes sur l'utilisation inappropriée des smartphones dans la vie commune publique et privée} Phubbing, ou l'utilisation inappropriée des smartphones dans la vie commune publique et privée. Ce mot-valise, formé des expressions anglaises phone et snubbing signifie le comportement répandu de s'occuper de son smartphone même en présence d'autres personnes. L'objectif de cette étude était de déterminer les causes de l'utilisation inappropriée des smartphones et d'examiner si le Phubbing entrave le comportement de communication et l'interaction sociale. A cet effet, un sondage en ligne a été mené auprès des étudiants du département informations de la HAW de Hambourg, afin de recueillir les expériences et les opinions des participants à propos du Phubbing. En outre, une étude observationnelle de situations réelles de Phubbing a été effectuée dans des lieux publics. Les conclusions de ces études empiriques ont servi de base pour un projet de groupe avec des participants sélectionnés de l'enquête en ligne, afin de discuter et d'analyser les résultats plus en profondeur. L'étude a été conduite pendant la période allant de novembre 2013 à mars 2014.

Descripteurs: enquête empirique, communication, téléphone, utilisation 
DOI 10.1515/iwp-2014-0052

\section{Einleitung}

Smartphones haben sich als fester Bestandteil der Gesellschaft etabliert und die Zahl der Nutzer ist in den vergangenen Jahren kontinuierlich gestiegen (vgl. Frees/van Eimeren 2013: 358). So kann der regelmäßige Griff zum Handy immer und überall geschehen - unbewusst und intuitiv. Mit der zunehmenden Verbreitung von Smartphones und der Verfügbarkeit des mobilen Internets wird der Anreiz für die Nutzer größer, sich auch dann mit ihren Geräten zu beschäftigen, wenn sie gerade mit anderen Personen zusammen sind. Dieses Phänomen hat, dank einer viralen Kampagne der australischen Werbeagentur McCann, einen Namen: Phubbing.

\section{Das Phänomen im Detail}

Als Teil einer Marketingstrategie initiierte die Agentur im Jahr 2012 das Projekt Stop Phubbing. Das offizielle Anliegen war es darauf hinzuweisen, dass die Welt zwar immer besser vernetzt wird, die Menschen sich aber im realen Leben voneinander entfernen. Bei dem Kunstwort Phubbing handelt es sich um ein Kofferwort, das aus den Begriffen phone und snubbing (Englisch für Telefon und jemanden vor den Kopf stoßen) zusammengesetzt ist. Die Kampagne umfasste die Homepage www.stopphubbing. com, eine Facebook-Seite, Plakate sowie diverse Markenartikel mit Slogans und Informationen über das unhöfliche Verhalten von Smartphone-Nutzern in Gesellschaft anderer Personen. Das Projekt löste eine weltweite Diskussion in Presse und Nachrichten über Phubbing aus und verbreitete sich innerhalb eines Jahres viral aus Australien in über 180 Länder (vgl. McCann 2013). In Deutschland griffen unter anderem das Nachrichtenportal heute.de (vgl. Krüger 2013) und die Internetseite der Süddeutschen Zeitung (vgl. Metallinos 2013) das Thema auf. Im Oktober 2013 wurde schließlich bekannt, dass hinter der Stop Phubbing Bewegung tatsächlich strategische Werbung für eine neue Ausgabe des MacquarieWörterbuchs steckt. McCann löste die Täuschung auf, indem die Agentur ein Video auf YouTube stellte, das den Prozess von der Erfindung des Wortes über die Verbreitung der Kampagne bis hin zu den Auswirkungen in den Medien zeigt (vgl. McCann 2013).

Auch wenn es sich bei der Stop-Phubbing-Bewegung um einen erfundenen Trend handelt, spricht der weltweite mediale Anklang für die Relevanz des Themas. Die
Vernachlässigung anderer Personen zugunsten der Smartphone-Nutzung ist somit als ein reales, gesellschaftliches Problem einzuordnen.

\section{Forschungsanspruch und Methodik}

Die breite Aufmerksamkeit, die das Thema Phubbing ausgelöst hat, lässt vermuten, dass auch das Forschungsinteresse in den kommenden Jahren weiter zunehmen wird. Die Studie mit dem Titel Phänomen Phubbing - Befragungen, Beobachtungen und Befunde zur unangebrachten Smartphone-Nutzung im privaten und öffentlichen Miteinander stellt eine entsprechende Grundlage dar. Die Thesis ist im Zeitraum von November bis März an der Hochschule für Angewandte Wissenschaften im Fach Medien und Information entstanden. Der primäre Untersuchungsanspruch bestand darin die Ursachen und Folgen des Phänomens mithilfe qualitativer und quantitativer Forschungsmethoden zu ermitteln. Dabei wird in Phubbing im öffentlichen und im privaten Miteinander unterschieden, wobei das persönliche Umfeld von Freunden, Familie und Bekannten im Fokus steht. In der Arbeit werden ergänzend der Hochschul- und Berufsalltag sowie die städtische Umgebung als öffentliche Räume für Phubbing aufgeführt. Die forschungsleitenden Fragen waren im Wesentlichen:

- In welchen spezifischen Situationen kommt Phubbing häufig vor?

- Welche Ursachen sind typisch für Phubbing?

- Beeinträchtigt Phubbing die Aufmerksamkeit und Kommunikationsfähigkeit des Smartphone-Nutzers?

- Wie wirkt sich Phubbing auf die zwischenmenschliche Beziehung zum Gegenüber aus?

Für den entsprechenden Erkenntnisgewinn wurden drei Forschungsmethoden angewendet. Den methodischen Schwerpunkt bildet eine Onlinebefragung unter den Studierenden des Departments Information an der HAW Hamburg. Insgesamt haben 125 Personen Angaben zu ihren persönlichen Erfahrungswerten und Meinungen zur unangebrachten Smartphone-Nutzung im Beisein anderer gemacht. Die Mehrheit der Befragten besitzt mit 118 Teilnehmern ein eigenes Smartphone, der Altersdurchschnitt liegt zwischen 18 und 29 Jahren. Die Umfrage wurde von einer parallel laufenden Beobachtungsstudie an öffentlichen Plätzen ergänzt, bei der reale Verhaltensweisen und Reaktionen im Zusammenhang mit Phubbing erfasst wurden. Demnach wurden Gruppen von zwei oder 
mehr Personen in einem Starbucks Coffee House und in der U-Bahn observiert, in denen eine unangebrachte Smartphone-Nutzung auftrat. Die so gewonnenen Erkenntnisse bildeten schließlich die Basis für das anschließende Fokusgruppenprojekt. Als Teilnehmer wurden fünf Studierende eingeladen, die sich zuvor an der Onlinebefragung beteiligt hatten. Ihnen wurden einzelne Befunde und Beobachtungen aus den vorangehenden Forschungsmethoden zur weiteren Diskussion und Analyse präsentiert. Das Gesamtergebnis aller Forschungsmethoden ist die Erfassung des Phänomen Phubbings unter Abbildung seiner Hintergründe und Auswirkungen auf das gesellschaftliche Miteinander im privaten wie öffentlichen Raum.

\section{Hintergrůinde und Ursachen}

Die bekannteste Umgebung für die unangebrachte Smartphone-Nutzung sind öffentliche Verkehrsmittel, die von 80 Prozent der Teilnehmer in der Onlinebefragung genannt werden. Darauf folgen Restaurants und Cafés sowie das private Zuhause, in denen 68 und 66 Prozent der Befragten Phubbing häufig erleben. Die Fokusgruppendiskussion ergab hierzu, dass Handybesitzer die Nutzung in diesen Umgebungen vom Alleinsein gewöhnt sind und das vertraute Verhalten darum auch in Gruppensituationen übertragen. Die am meisten genutzten Funktionen während des Phubbings sind digitale Kommunikationsdienste wie Chat-, E-Mail- und MessengerProgramme. Auch Social Media sind beliebte Apps, die in Gesellschaft anderer Personen verwendet werden (vgl. Abb. 1).

Eine Studentin erklärt diese Ergebnisse in der Fokusgruppe wie folgt: „In dem Moment wo du deine ganzen

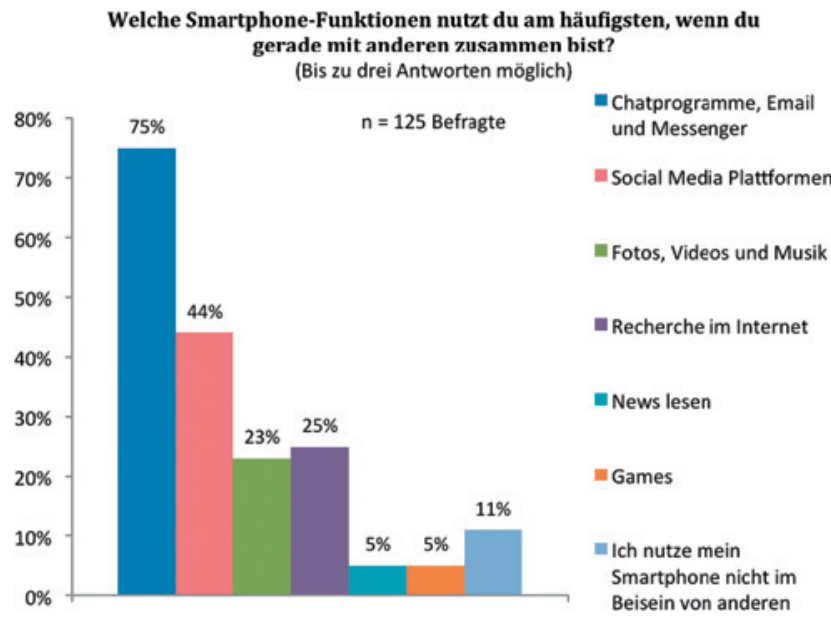

Abb. 1: Funktionsnutzung während des Phubbings.
Programme öffnest und auch über Facebook kommunizierst, dann hast du einfach eine ganz andere Reichweite. Dann wissen viel mehr Menschen was du machst und wo du bist. Das ist ein extremes Mittelungsbedürfnis.“ Die Smartphone-Besitzer sind also über diese Apps dauerhaft für andere erreichbar und es gibt entsprechend zahlreiche Anstöße, diese Anwendungen aufzurufen. Über digitale Kommunikationsdienste und soziale Medien können Nutzer ihre Gedanken mit einer breiteren Masse teilen, als mit dem aktuellen Gegenüber. Die Beschäftigung mit dem Gerät dauert im Durchschnitt 1:07 Minuten. Dies ergab die im Rahmen der Forschung durchgeführte Beobachtungsstudie, bei der insgesamt acht Phubbing-Situationen in der U-Bahn und zwölf in einem Café observiert wurden. Aus den Erkenntnissen der Studie geht außerdem hervor, dass sich SmartphoneBesitzer länger mit dem Handy beschäftigen, wenn es zuvor geklingelt hat, weil sie dann oft nicht nur auf die eingegangene Nachricht reagieren, sondern die Gelegenheit nutzen, auch andere Apps auf Neuigkeiten überprüfen.

Die konkreten Gründe für Phubbing im privaten Miteinander können je nach Situation variieren und hängen auch mit der persönlichen Einstellung des SmartphoneBesitzers zusammen. Als ausschlaggebende Faktoren nennen die Teilnehmer der Onlinebefragung:

- Nachrichten und Push-Mitteilungen (36 Prozent)

- Angewohnheit, häufig auf das Handy zu schauen (32 Prozent)

- Angst davor, etwas zu verpassen (27 Prozent)

- ein erhöhtes Mitteilungs- oder Selbstdarstellungsbedürfnis über Apps (mehrfach genannt in der Fokusgruppendiskussion)

In Einzelfällen kann die Smartphone-Nutzung auch ein Zeichen für das Desinteresse am Gegenüber oder ein Ventil für Ärger oder Unwohlsein darstellen. Ergänzend hierzu ist festzuhalten, dass der Griff zum Handy unbewusst und automatisch erfolgen kann, so dass sich der „Phubber“ seines Verhaltens im privaten Miteinander nicht immer direkt bewusst ist (vgl. Abb. 2).

Auch im öffentlichen Raum sind die Motive für die unangebrachte Smartphone-Nutzung vielfältig. Im Hochschulalltag beschäftigen sich Studierende meist dann mit ihren Handys, wenn sie sich nicht für die Studieninhalte interessieren. Im Frontalunterricht von Vorlesungen geschieht das eher als in Kursen, in denen die Studierenden aktiv mitarbeiten müssen. Im Berufsleben kommt es hingegen auf die Branche an, ob Smartphones eine Rolle während der Arbeitszeit spielen. So kann die regelmäßige Smartphone-Nutzung ein fester Bestandteil der Arbeit bestimmter Berufsgruppen sein, in denen die Arbeitneh- 
Ich vermute, dass der regelmäßige Griff zum Smartphone automatisch und unbewusst erfolgt.

$n=125$ Befragte

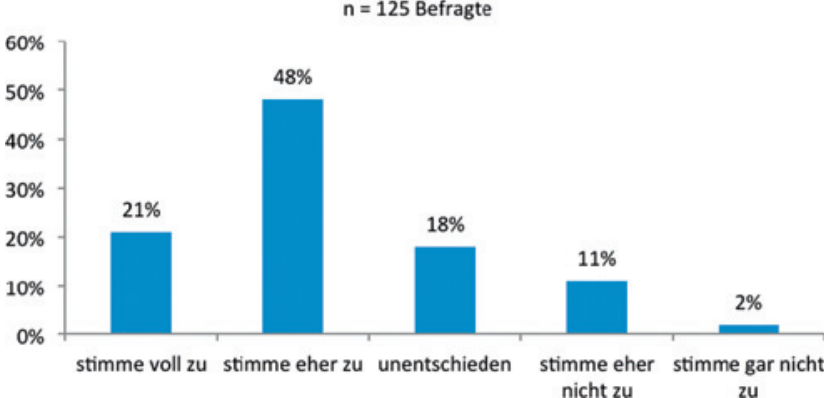

Abb. 2: Bewusstsein für den Griff zum Smartphone.

mer stets über aktuelle Trends, Entwicklungen und Neuigkeiten informiert sein müssen. Eine andere Studentin nennt in der Fokusgruppendiskussion einige Beispiele: „Im Journalismus, im Medienbereich und speziell im Social Media-Bereich ist das [Phubbing] eigentlich schon fast ein Muss.“ Im Straßenbild ist Phubbing vor allem auf drei Motive zurückzuführen:

- dauerhafte Erreichbarkeit

- Zeitersparnis

- Langeweile und fehlende Beschäftigungsalternativen

Über verschiedene Apps können Smartphone-Nutzer auch in diesem Umfeld zu jeder Zeit Mitteilungen erhalten. Wer gerade als Fußgänger im Straßenverkehr unterwegs ist, wird so gegebenenfalls animiert, sich in dieser Umgebung mit neuen Nachrichten und Funktionsänderungen zu beschäftigen, um dies beim Erreichen des Zielorts bereits erledigt zu haben. Die Erkenntnis, dass Phubbing im Straßenverkehr häufig zur Ablenkung bei Langeweile vorkommt, verdeutlicht die Rolle von Smartphones als intuitive Beschäftigungsmittel.

\section{Auswirkungen und Risiken}

Im privaten Miteinander wird die Smartphone-Nutzung allgemein als ein eher unangebrachtes Verhalten bewertet. In den konkreten Phubbing-Situationen kommt es jedoch auf die subjektive Wahrnehmung des Einzelnen an, ob ihn das Verhalten seines Gegenübers stört. Darauf nehmen besonders die nachfolgenden Kriterien Einfluss:

- der Grund für die Smartphone-Nutzung

- die Dauer und Häufigkeit des Phubbings

- das persönliche Verhältnis zum Gegenüber

- die Gruppengröße, in der das Phubbing vorkommt

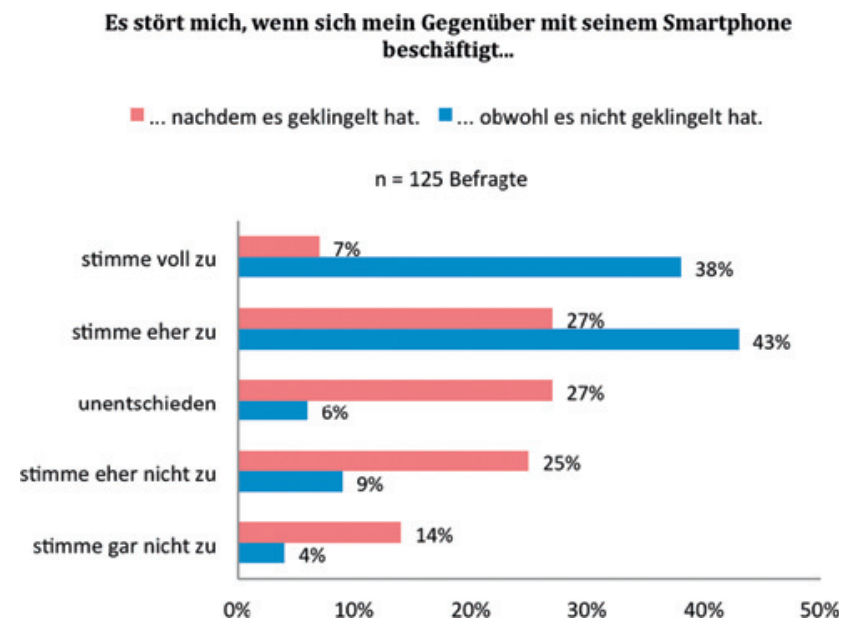

Abb. 3: Störfaktor Phubbing mit und ohne Klingelton.

Auffällig ist hierbei, dass Personen, die selbst einen großen Teil ihrer Freizeit im Internet verbringen, oftmals zu einer höheren Akzeptanz gegenüber Phubbing neigen als die Befragten, die im Vergleich eine kürzere Zeit online sind. Wird der Griff zum Handy vorher angekündigt und begründet, schafft das grundsätzlich ein größeres Verständnis für das Verhalten des Handy-Besitzers. Aus den Umfrageergebnissen geht außerdem hervor, dass Betroffene deutlich toleranter auf die Smartphone-Nutzung ihres Gegenübers reagieren, wenn dessen Handy zuvor geklingelt hat.

Weiterhin wird häufiges Phubbing als störender empfunden als eine einmalige Smartphone-Nutzung, die länger dauert. Auch die zwischenmenschliche Beziehung zum Gegenüber und die jeweilige Situation beeinflussen die Wahrnehmung der Betroffenen. In persönlichen Unterhaltungen $\mathrm{zu}$ zweit wird Phubbing kritischer angesehen als in größeren Gruppen. Ein Fokusgruppenteilnehmer erläutert hierzu: „Wenn man in einer größeren Gruppe ist, stört es mich nicht. [...] Dann hast du ja noch andere Bezugspersonen mit denen du reden kannst und Augenkontakt hast." Folglich fühlen sich betroffene Personen während der Smartphone-Nutzung ihres Gegenübers weniger vernachlässigt, sobald alternative Gesprächspartner anwesend sind.

76 Prozent der Smartphone-Nutzer gehen davon aus, dass Phubbing im privaten Miteinander $\mathrm{zu}$ einem Aufmerksamkeitsverlust des Smartphone-Nutzers gegenüber seiner Begleitung führen kann. Je nach Situation und Wahrnehmung des Einzelnen kann dies als Zeichen von Respektlosigkeit und Desinteresse aufgefasst werden, was ein Gefühl der Vernachlässigung auslöst. Aus diesem Empfinden leiten sich auch die jeweiligen Reaktionen der Betroffenen ab. In vielen Fällen warten die Begleitungen das Phubbing ihres Gegenübers ab, bis dieser 
Smartphone-Nutzer sind weniger aufmerksam und erledigen ihre Tătigkeiten weniger effektiv...

[... während des Unterrichts. = ... während der Arbeitszeit.

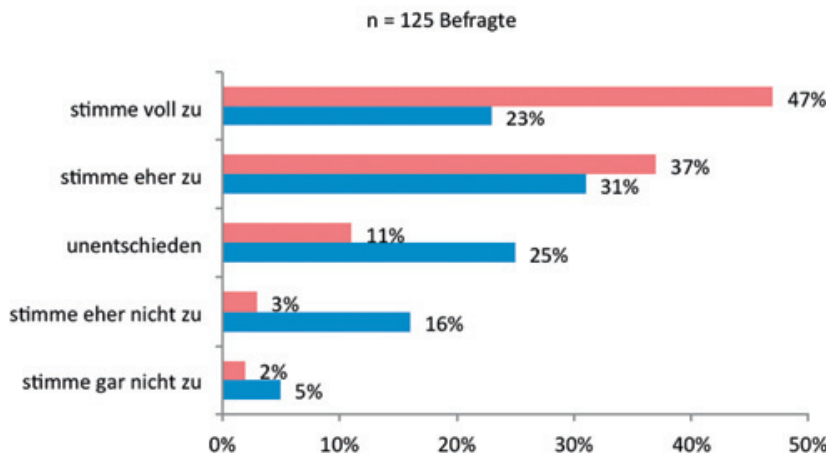

Abb. 4: Aufmerksamkeitsverlust während des Unterrichts und der Arbeitszeit durch Phubbing.

sein Smartphone wieder weglegt (47 Prozent). Gelegentlich wird das Verhalten auch gespiegelt und sie greifen ihrerseits zum Handy (27 Prozent). Sind sie jedoch sehr über das Phubbing verärgert, sind auch eine offene Konfrontation des Smartphone-Nutzers oder ein direkter Gesprächsabbruch möglich (18 Prozent).

Sowohl im Hochschul- als auch im Berufsalltag kann die Smartphone-Nutzung einen schlechten Eindruck bei Dozenten oder Vorgesetzten hinterlassen. Auch hier kommt es darauf an, zu welchem Zweck das Handy verwendet wird und wie lange die Nutzung dauert. In Vorlesungen und Seminaren ist die Gefahr der Unaufmerksamkeit besonders hoch, wenn sich die Studierenden bewusst dafür entscheiden dem Unterricht zugunsten des Phubbings nicht mehr zu folgen. Das Risiko, dass Aufgaben nicht so effektiv erledigt werden, wird am Arbeitsplatz meist als geringer eingeschätzt, wie Abbildung 4 veranschaulicht.

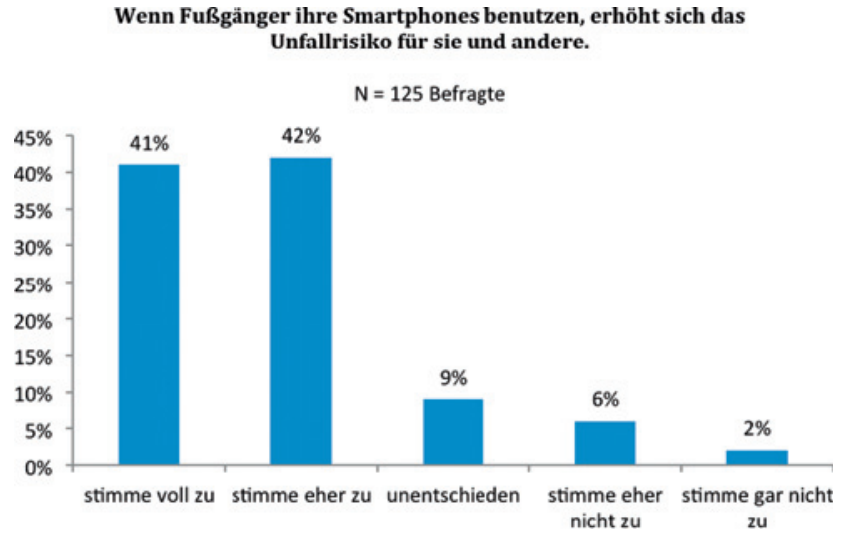

Abb. 5: Erhöhung des Unfallrisikos durch Phubbing.
Je nach Branche kann die Smartphone-Nutzung so in den Berufsalltag integriert werden, dass der Arbeitsablauf nicht darunter leidet. Das lässt sich beispielsweise bei Bürotätigkeiten am Computer leichter umsetzen als in Dienstleistungsberufen bei denen Kunden betreut und beraten werden müssen. Im Straßenverkehr ist hingegen eindeutig mit einem Aufmerksamkeitsverlust zu rechnen, wenn Fußgänger in dieser Umgebung ihr Smartphone verwenden. Die so erhöhte Unfallgefahr ist aus Sicht der Mehrheit aller 125 befragten Studierenden ein wesentlicher Risikofaktor von Phubbing (vgl. Abb. 5).

\section{Ausblick}

Phubbing ist ein bekanntes und kritisiertes Phänomen unserer modernen Gesellschaft. Auch wenn Betroffene das Verhalten bei ihrem Gegenüber bemängeln, tritt die unangebrachte Smartphone-Nutzung dennoch immer wieder auf. Da Smartphones zunehmend Einzug sowohl in den privaten als auch in den beruflichen Alltag halten, ist davon auszugehen, dass sich Phubbing in $\mathrm{Zu}$ kunft noch stärker verbreiten wird. Die Ergebnisse der Studie zeigen, dass die Verhaltensweise verschiedene Ursachen haben kann und durchaus Risiken mit sich bringt. So kann Phubbing die Kommunikationsfähigkeit und das gesellschaftliche Miteinander im privaten wie öffentlichen Raum beeinträchtigen. Im Straßenverkehr stellt die Smartphone-Nutzung definitiv eine Kommunikationsbarriere dar und ist als risikotragendes Verhalten $\mathrm{zu}$ betrachten.

\section{Literatur}

Frees, Beate; van Eimeren, Birgit: Ergebnisse der ARD/ZDF-Onlinestudie: Rasanter Anstieg des Internetkonsums - Onliner fast drei Stunden täglich im Netz. In: Media Perspektiven, Nr. 7-8, 2013, S. 358

Klein, Vanessa: Phänomen Phubbing: Befragungen, Beobachtungen und Befunde zur unangebrachten Smartphone-Nutzung im privaten und öffentlichen Miteinander. Hamburg, Hochschule für Angewandte Wissenschaften, FB Medien und Information, Bachelorarbeit, 2014.

Krüger, Alfred: Kampagne gegen Smartphone-Unsitten: Phubbing: Wenn der Partner nur noch auf das Handy guckt [online]. In: heute.de - http://www.heute.de/PhubbingWenn-der-Partner-nur-noch-auf-das-Handy-guckt-29294712. html [2014-03-13]

McCANN: Phubbing: A Word is Born [Video]. In: Phubbing: A Word is Born, YouTube - https://www.youtube.com/watch?v=ZSOfuUYCV_0 [2014-03-12] 
Metallinos, Christina: Kampagne gegen „Phubbing“: Leute, die auf Handys starren [online]. In: Süddeutsche.de - http://www. sueddeutsche.de/leben/kampagne-gegen-phubbing-leute-dieauf-handys-starren-1.1742662 [2014-03-13]

Stop Phubbing: Stop Phubbing [online]. In: Stop Phubbing - http:// stopphubbing.com/ [2014-03-13]

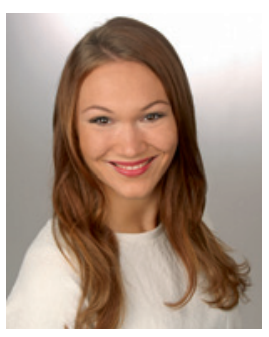

\section{Vanessa Klein}

HAW Hamburg

Medien und Information (B.A.)

Otzenstraße 1

22767 Hamburg

vanessa.klein@haw-hamburg,de

Vanessa Klein wurde 1989 in Hameln geboren und schloss die Schule 2009 am Schiller-Gymnasium Hameln mit dem Abitur ab. Anschließend zog sie für das Studium nach Hamburg und absolvierte im Juni 2014 den Bachelor of Arts im Fach Medien und Information an der Hochschule für Angewandte Wissenschaften Hamburg. Aktuell studiert sie an der HAW Hamburg den Master Medien, Information, Bibliothek. 